\title{
Steady, barotropic wind and boundary-driven circulation on a polar plane
}

\author{
ANDREW J. WILLMOTT †* and MARIA LUNEVA $\dagger$ \\ $\dagger$ National Oceanography Centre, 6 Brownlow Street, Liverpool, L3 5DA, UK \\ †School of Marine Science and Technology, Newcastle University, NE1 7RU, UK \\ (Received 12 December 2013; in final form 24 November 2014; first published online ????)
}

\begin{abstract}
Steady, linear, barotropic wind and boundary forced circulation solutions in the presence of linear bottom friction are analytically derived in a circular basin of uniform depth on a polar tangent plane in which only first order effects of the Earth's curvature are retained. Approximate solutions are constructed by using the well known method of aggregating the interior inviscid Sverdrup balance solution and the frictional wall boundary layer solution. In contrast to the width of mid-latitude frictional western boundary layers that scale as $\mathrm{O}(\mu / f)$, the width of the polar frictional boundary layer adjacent to the basin wall is wider, scaling as $\mathrm{O}\left((\mu / f)^{1 / 2}\right)$, where $\mu$ is the bottom friction coefficient, $f$ is the coriolis parameter. Solutions are presented for a variety of wind stress curl distributions and for a prescribed inflow/outflow representative of the exchange of water masses between the Arctic and Atlantic basins. Boundary forced solutions are also derived in a basin with a uniform width step shelf. For this basin geometry the flow is mainly confined to the shelf, although a parameter regime is identified that supports significant flow in the deep basin.
\end{abstract}

Keywords: Arctic Ocean circulation; Polar plane

\section{Introduction}

What are the dominant processes that control the ocean circulation of the Arctic? The answer to this question has acquired new urgency with the well documented retreat of Arctic summer sea ice during the $21^{\text {st }}$ Century (Wang and Overland 2009) and the concomitant impacts on Arctic Ocean ecosystems and the socio-economics of the indigenous peoples of the region. Using a coupled numerical sea ice- barotropic ocean circulation model, Proshutinsky and Johnson $(1997,2001)$ have revealed the importance of wind-driving in determining the Arctic Ocean circulation and its sea ice dynamics. These studies demonstrate that the circulation switches between an anticyclonic regime, characterised by a well-defined Beaufort Gyre located in the Canadian basin of the western Arctic, and a cyclonic regime. Each of these regimes lasts typically 4 to 8 years, leading to an oscillatory mode with period of 8 to 16 years. In the anticyclonic regime the surface Ekman transport associated with the Beaufort Gyre is directed towards the gyre interior (Proshutinsky et al. 2009) which acts to trap surface melt waters within the gyre. This regime is also characterised by a strong transpolar drift that advects sea ice out of the Arctic.

As the complexity of coupled numerical sea ice-ocean circulation models increases, the interpretation of the model results becomes more challenging. It is well recognised that ocean process models provide an invaluable independent tool for interpreting the results from complex ocean circulation models. Interestingly, there is a conspicuous lack of ocean process models developed for Arctic applications due mainly to the fact that on the polar plane (the high-latitude equivalent of the mid-latitude $\beta$-plane) the meridional gradient of the Coriolis

*Corresponding author. Email: andrew.willmott@ncl.ac.uk 
parameter varies linearly with latitude, which produces an added complexity in the analysis of the shallow water equations.

Planetary (Rossby) wave dynamics in a circular polar basin were considered by LeBlond (1964) where the "polar plane approximation" was developed. The polar plane approximation differs from the mid-latitude $\beta$-plane approximation in that the derivative of the Coriolis parameter is a linear function of the radial distance to the pole. Harlander (2005) extended the analysis of LeBlond (1964) by studying planetary wave dynamics on the delta plane. This latter approximation reduces to the polar plane when the origin of the delta plane lies at the geographical pole. The topic of geostrophic adjustment in a polar basin has been treated analytically and numerically on the polar plane by Luneva et al. (2012). Steady state barotropic circulation in a polar circular basin driven by prescribed inflow and outflow is treated analytically and experimentally by Imawaki and Takano (1974). The authors compare the solutions on a polar-cap with those in a uniformaly rotating basin. Again, in the spirit of simplified models, Nøst and Isachsen (2003) develop a diagnostic model for steady large-scale circulation in the Nordic Seas and the Arctic Ocean that incorporates realistic topography.

The purpose of this study is to present new analytical solutions for wind and boundary driven steady-state barotropic ocean circulation in a circular Arctic basin located on a polar plane. Section 2 develops the governing equations; wind-driven solutions in a flat bottom basin are presented in section 3; boundary forced solutions, associated with inflow/outflow, are derived in section 4 for a flat bottom basin; section 5 extends the solutions in the previous section with the inclusion of step shelf of uniform width; the paper concludes with a summary and discussion of future research in section 6 .

\section{Governing equations}

The starting point for the analysis of wind-driven, frictionally modified barotropic circulation in a polar basin of uniform depth $H$ are the linearised shallow water equations on a tangent plane to the Earth at the pole:

$$
\begin{aligned}
& \frac{\partial u}{\partial t}-f v=-g \cos \theta \frac{\partial \eta}{\partial r}-\mu u+\frac{\tau^{r}}{\rho H}, \\
& \frac{\partial v}{\partial t}+f u=-\frac{g}{r} \frac{\partial \eta}{\partial \phi}-\mu v+\frac{\tau^{\phi}}{\rho H}, \\
& \frac{\partial \eta}{\partial t}+\frac{\cos \theta}{r} \frac{\partial}{\partial r}(H r u)+\frac{1}{r} \frac{\partial}{\partial \phi}(H v)=0 .
\end{aligned}
$$

In (1) and (2) $r, \phi$ are polar coordinates on a tangent plane to the Earth at the pole with origin located on the rotational axis of the Earth; $u, v$ are the velocity components in the radial and azimuthal direction, respectively; $\mu$ is the coefficient of Rayleigh friction; $\rho$ is the constant density of the ocean; $\tau^{r}, \tau^{\phi}$ are the wind stress components in the radial and azimuthal directions, respectively; $\eta$ is the free surface displacement; $g$ is the gravitational acceleration; $f=2 \Omega \cos \theta$ is the Coriolis parameter, where $\theta$ is the co-latitude of any point on the tangent plane. Figure 1 shows a schematic of the polar tangent plane. Following LeBlond (1964) the radial gradient of $f$ can be readily calculated as follows. Provided the departure of the tangent plane from the surface of the Earth is not too large

$$
r=R \sin \theta,
$$

where $R$ is the radius of the Earth. Then on using (3), we obtain

$$
\frac{\mathrm{d} f}{\mathrm{~d} r}=-2 \Omega \sin \theta \frac{\mathrm{d} \theta}{\mathrm{d} r}=-2 \Omega \frac{\sin \theta}{R \cos \theta}=\frac{2 \Omega r}{R^{2} \cos \theta} .
$$


Upon neglecting terms $\mathrm{O}\left((r / R)^{2}\right)$, we arrive at the polar plane approximation

$$
\frac{\mathrm{d} f}{\mathrm{~d} r}=-\frac{2 \Omega r}{R^{2}}
$$

first discussed by LeBlond (1964).

In sections 3 and 4 we will neglect topography and impose the rigid-lid approximation, in which case (2) permits a velocity streamfunction $\Psi$ defined as

$$
r u=-\frac{\partial \Psi}{\partial \phi}, \quad \frac{v}{\cos \theta}=\frac{\partial \Psi}{\partial r},
$$

where we note that $\theta=\theta(r)$. Using (1) and (5) it is then straightforward to obtain the unsteady vorticity equation in terms of $\Psi$ :

$$
r \nabla^{2} \frac{\partial \Psi}{\partial t}+\mu r \nabla^{2} \Psi+\frac{r f}{R^{2}} \frac{\partial \Psi}{\partial \phi}=\cos \theta \frac{\partial}{\partial r}\left(\frac{r \tau^{\phi}}{\rho H}\right)-\frac{\partial}{\partial \phi}\left(\frac{\tau^{r}}{\rho H}\right)
$$

On the polar plane we approximate $\cos \theta$ by unity in (6), in which case the right-hand side of this equation is clearly

$$
r \boldsymbol{k} \cdot \nabla \times\left(\frac{\boldsymbol{\tau}}{\rho H}\right)
$$

where the operator

$$
r \nabla^{2} \equiv \frac{\partial}{\partial r}\left(r \frac{\partial}{\partial r}\right)+\frac{1}{r} \frac{\partial^{2}}{\partial \phi^{2}}, \quad \text { and } \quad \boldsymbol{k} \cdot \boldsymbol{\nabla} \times \boldsymbol{\tau}=\frac{1}{r}\left(\frac{\partial}{\partial r}\left(r \tau^{\phi}\right)-\frac{\partial \tau^{r}}{\partial \phi}\right) .
$$

In a steady-state, (6) reduces to the polar Sverdrup balance for the interior streamfunction

$$
\frac{f}{R^{2}} \frac{\partial \Psi}{\partial \phi}=\boldsymbol{k} \cdot \boldsymbol{\nabla} \times\left(\frac{\boldsymbol{\tau}}{\rho H}\right)
$$

valid away from rigid boundaries where frictional boundary layers are, in general, required to satisfy the "no-normal flow" boundary condition.

In the following sections of this paper we obtain solutions of the steady version of (6) in a circular basin of radius a, centred at the pole, for a variety of idealised wind stress fields. We will also obtain solutions of this equation which are driven by a prescribed streamfunction on $r=a$ which represents inflows and outflows across the boundary. Clearly, the wind and boundary forced solutions can be superposed to obtain forced solutions that are representative of a prototype barotropic Arctic Ocean circulation.

\section{Wind forced solutions}

In this section we obtain solutions of

$$
\mu r \nabla^{2} \Psi+\frac{r f}{R^{2}} \frac{\partial \Psi}{\partial \phi}=r \boldsymbol{k} \cdot \nabla \times\left(\frac{\boldsymbol{\tau}}{\rho H}\right)
$$

subject to

$$
\Psi=0 \quad \text { on } \quad r=a,
$$

together with the requirement that $\Psi$ is $2 \pi$-periodic and bounded everywhere in the circular domain. 


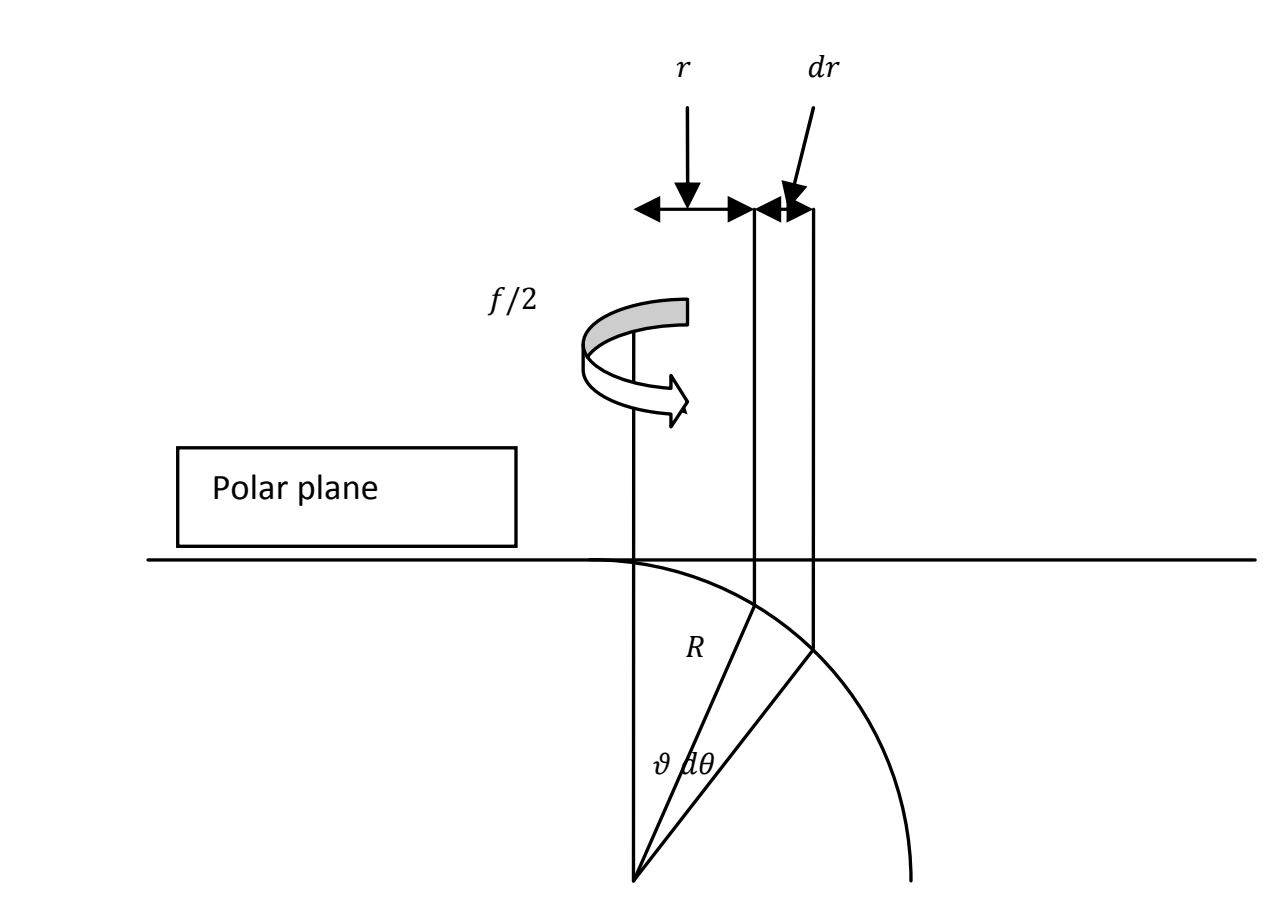

Figure 1. Schematic of the polar plane.

Polar plane

$\rightarrow$

Fic 


\subsection{Radially symmetric wind stress}

Consider the azimuthal wind stress

$$
\tau^{r}=0, \quad \tau^{\phi}=W(r) .
$$

Clearly, (8) admits radially-symmetric solutions $\Psi=\Psi(r)$, where

$$
\mu \frac{\mathrm{d}}{\mathrm{d} r}\left(r \frac{\mathrm{d} \Psi}{\mathrm{d} r}\right)=\frac{\mathrm{d}}{\mathrm{d} r}\left(\frac{r W}{\rho H}\right) .
$$

Integrating this equation, and demanding that $\Psi$ is bounded in the domain, we obtain

$$
\mu \frac{\mathrm{d} \Psi}{\mathrm{d} r}=\frac{W}{\rho h} \quad \Longrightarrow \quad v=\frac{W}{\rho h} .
$$

The velocity field (11b) is dependent upon the existence of bottom friction. It is therefore reasonable to enquire whether an "interior" inviscid solution $\Psi_{I}$ can be determined. From (8) we see that

$$
\frac{r f}{R^{2}} \frac{\partial \Psi_{I}}{\partial \phi}=\frac{\mathrm{d}}{\mathrm{d} r}\left(\frac{r W}{\rho H}\right)
$$

which upon integration with respect to $\phi$ yields

$$
\Psi_{I}=\frac{\phi R^{2}}{r f} \frac{\mathrm{d}}{\mathrm{d} r}\left(\frac{r W}{\rho H}\right)
$$

The form of $W$ determines whether the circulation is bounded in the domain. In general (12) does not satisfy (9) and a frictional boundary layer adjacent to $r=a$ is required to satisfy this boundary condition. Indeed, the structure of this boundary layer is presented in section 3.2. It suffices to note at this stage that a $2 \pi$-periodic boundary layer solution that matches (12) is not possible. Thus, the steady frictionally modified solution of (8) for an radially-symmetric wind stress is itself radially-symmetric.

Typical forms of $W$ relevant to the Arctic include

$$
W=\tau_{0} \widehat{r}^{\beta} \mathrm{e}^{-\alpha r},
$$

and

$$
W= \begin{cases}\tau_{0} & 0 \leq r \leq b, \\ \tau_{0}(b / r)^{\gamma} & b \leq r \leq a,\end{cases}
$$

where $\tau_{0}$ is the magnitude of the wind stress, $\widehat{r}=r / a$ and $\alpha, \beta, \gamma \geq 0$, are constants. It is straightforward to calculate $\Psi$ for either forms of (13) and the details are left for the reader.

\subsection{Anti-symmetric wind stress curl}

We consider a wind stress curl of the form

$$
\mathrm{F}(r, \phi)=\boldsymbol{k} \cdot \boldsymbol{\nabla} \times\left(\frac{\boldsymbol{\tau}}{\rho H}\right)=\frac{1}{\rho H a} W(r) \sin \phi,
$$

where $W$ is given by (13a). More specifically, setting $\alpha=0, \beta=1$ the wind stress curl becomes

$$
\mathrm{F}(r, \phi)=\frac{\tau_{0}}{\rho H a^{2}} r \sin \phi,
$$

whence the interior inviscid Sverdrup balance becomes

$$
\frac{f}{R^{2}} \frac{\partial \Psi_{I}}{\partial \phi}=\frac{\tau_{0}}{\rho H a^{2}} r \sin \phi
$$


Hereafter, we treat $f$ as constant in (15). By inspection of (14) it is clear that the contours of the wind stress curl are a family of straight parallel lines of zonal orientation and that $F$ is positive (negative) for $0<\phi<\pi(-\pi<\phi<0)$. Integrating (15) we find that the bounded interior solution is given by

$$
\Psi_{I}=-\frac{\tau_{0} R^{2}}{\rho f H a^{2}} \cos \phi .
$$

The streamlines are a family of straight lines all parallel to the $y$-axis, i.e., oriented meridionally. A frictional boundary layer adjacent to $r=a$ is required to satisfy the boundary condition (9). We therefore let the total solution in the circular domain take the form

$$
\Psi=\Psi_{I}+\widehat{\Psi}(s, \phi)
$$

where $\widehat{\Psi}$ is the boundary layer streamfunction and

$$
s=(a-r) /(\mu / f)^{p},
$$

is the stretched boundary layer radial coordinate, with the constant $p>0$ to be determined and $(\mu / f) \ll 1$. Substituting (17) into (8) and employing (15) we find that the leading order boundary layer equation is given by

$$
\frac{\partial^{2} \widehat{\Psi}}{\partial s^{2}}+\frac{1}{R^{2}} \frac{\partial \widehat{\Psi}}{\partial \phi}=0
$$

provided that $p=1 / 2$. The size of the largest terms neglected in deriving the boundary layer equation (18) are $\mathrm{O}\left((\mu / f)^{1 / 2}\right)$. In contrast to the width of the mid-latitude frictional western boundary layer, which scales as $\mu / f$, the boundary layer width in the polar basin is wider, scaling as $(\mu / f)^{1 / 2}$. The boundary conditions for (18) are

$$
\begin{aligned}
& \widehat{\Psi}=-\Psi_{I}(a, \phi) \quad \text { on } \quad s=0, \\
& \widehat{\Psi} \rightarrow 0 \quad \text { as } s \rightarrow \infty \text {. }
\end{aligned}
$$

We seek periodic solutions of (18) of the form

$$
\widehat{\Psi}=\sum_{n=-\infty}^{\infty} \mathrm{e}^{\mathrm{i} n \phi} g_{n}(s), \quad \text { where } \quad \frac{\mathrm{d}^{2} g_{n}}{\mathrm{~d} s^{2}}+\frac{\mathrm{i} n}{R^{2}} g_{n}=0 .
$$

The solution for $g_{n}$ satisfying $g_{n} \rightarrow 0$, as $s \rightarrow \infty$, is given by

$$
g_{n}(s)= \begin{cases}a_{n} \exp [-(s / R) \sqrt{n / 2}(1-\mathrm{i})], & n>0, \\ a_{n} \exp [-(s / R) \sqrt{|n| / 2}(1+\mathrm{i})], & n<0 .\end{cases}
$$

Using (19b) it is clear that $g_{0}(s) \equiv 0$. Applying (19a) we obtain

$$
\sum_{n=-\infty}^{-1} a_{n} \mathrm{e}^{\mathrm{i} n \phi}+\sum_{n=1}^{\infty} a_{n} \mathrm{e}^{\mathrm{i} n \phi}=\frac{\tau_{0} R^{2}}{2 \rho f H a}\left[\mathrm{e}^{\mathrm{i} \phi}+\mathrm{e}^{-\mathrm{i} \phi}\right],
$$

whence

$$
a_{-1}=a_{1}=\frac{\tau_{0} R^{2}}{2 \rho f H a},
$$

with $a_{n}=0$ otherwise. Thus we have

$$
\Psi=\frac{\tau_{0} R^{2}}{2 \rho f H a^{2}}\left\{-r \cos \phi+a \exp \left[-\frac{s}{\sqrt{2} R}\right] \cos \left[\phi+\frac{s}{\sqrt{2} R}\right]\right\} .
$$


(a) $\mu=10^{-7} \mathrm{~S}^{-1}$

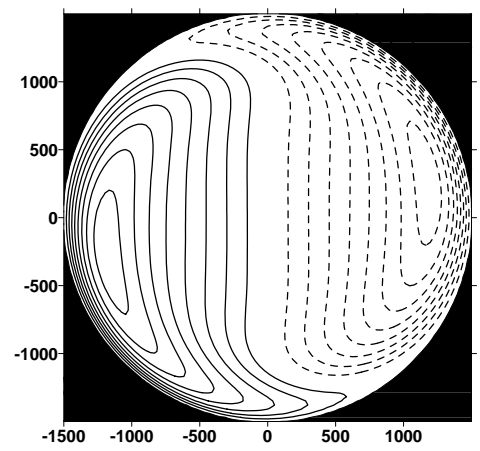

(b) $\mu=10^{-6} \mathrm{~s}^{-1}$

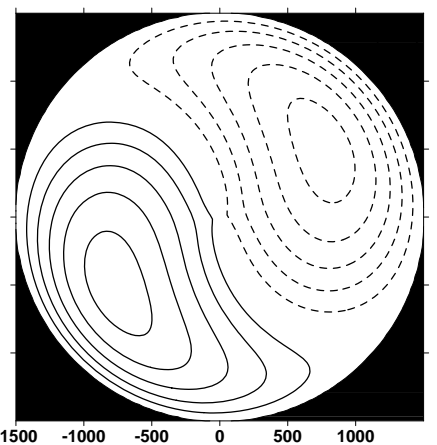

(c) $\mu=10^{-5} \mathrm{~s}^{-1}$

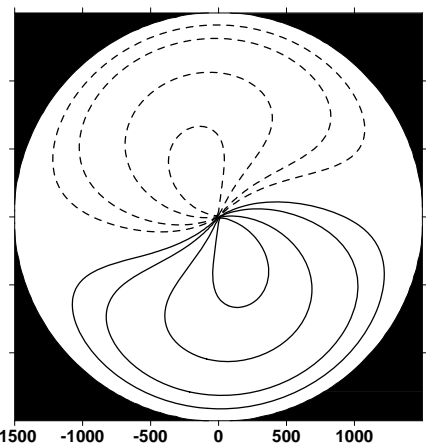

Figure 2. Contour plots of the dimensionless velocity streamfunction (20) for various values of the bottom friction parameter $\mu$. Contour interval is 0.1 . (a) $\mu=10^{-7} \mathrm{~s}^{-1}$; (a) $\mu=10^{-6} \mathrm{~s}^{-1}$; (a) $\mu=10^{-5} \mathrm{~s}^{-1}$.

\begin{tabular}{|c|c|}
\hline Parameter & Value \\
\hline$R$, radius of the Earth & $6371 \mathrm{~km}$ \\
\hline$a$, radius of the basin & $1500 \mathrm{~km}$ \\
\hline$\rho$ & $1000 \mathrm{~kg} \mathrm{~m}^{-3}$ \\
\hline$\tau_{0}$ & $0.1 \mathrm{~kg} \mathrm{~m}^{-1} \mathrm{~s}^{-2}$ \\
\hline$H$ & $4000 \mathrm{~m}$ \\
\hline$\widehat{\phi}$ & $10.1^{\circ}$ \\
\hline
\end{tabular}

Table 1. Parameter values used throughout this paper

Figure 2 shows contours of the non-dimensional form of (20) when $\mu=10^{-7} \mathrm{~s}^{-1}, 10^{-6} \mathrm{~s}^{-1}$ and $10^{-5} \mathrm{~s}^{-1}$ with other parameter values listed in Table 1 . The streamfunction is nondimensionalised by $\Psi_{0}=\tau_{0} R^{2} /\left(\rho f H a^{2}\right)$. Negative values of the streamfunction are contoured using dashed lines in figure 2 and all contour plots shown hereafter. In figure 2(a) the inviscid interior solution occupies the majority of the domain and is characterised by meridionally aligned flow as predicted by (16). As dissipation increases figures $2(b, c)$ reveal that the two gyres rotate in a counter clockwise sense and the flow is everywhere influenced by bottom friction. It is also instructive to compare (20) with the equivalent $f$-plane solution, the details of which are given in the Appendix.

For fixed $\phi,(14)$ increases monotonically with $r$. In contrast, consider the azimuthal wind stress

$$
\tau^{r}=0, \quad \tau^{\phi}=\tau_{0} \widehat{r}^{2} \mathrm{e}^{-\alpha r} \sin \phi .
$$


(a)
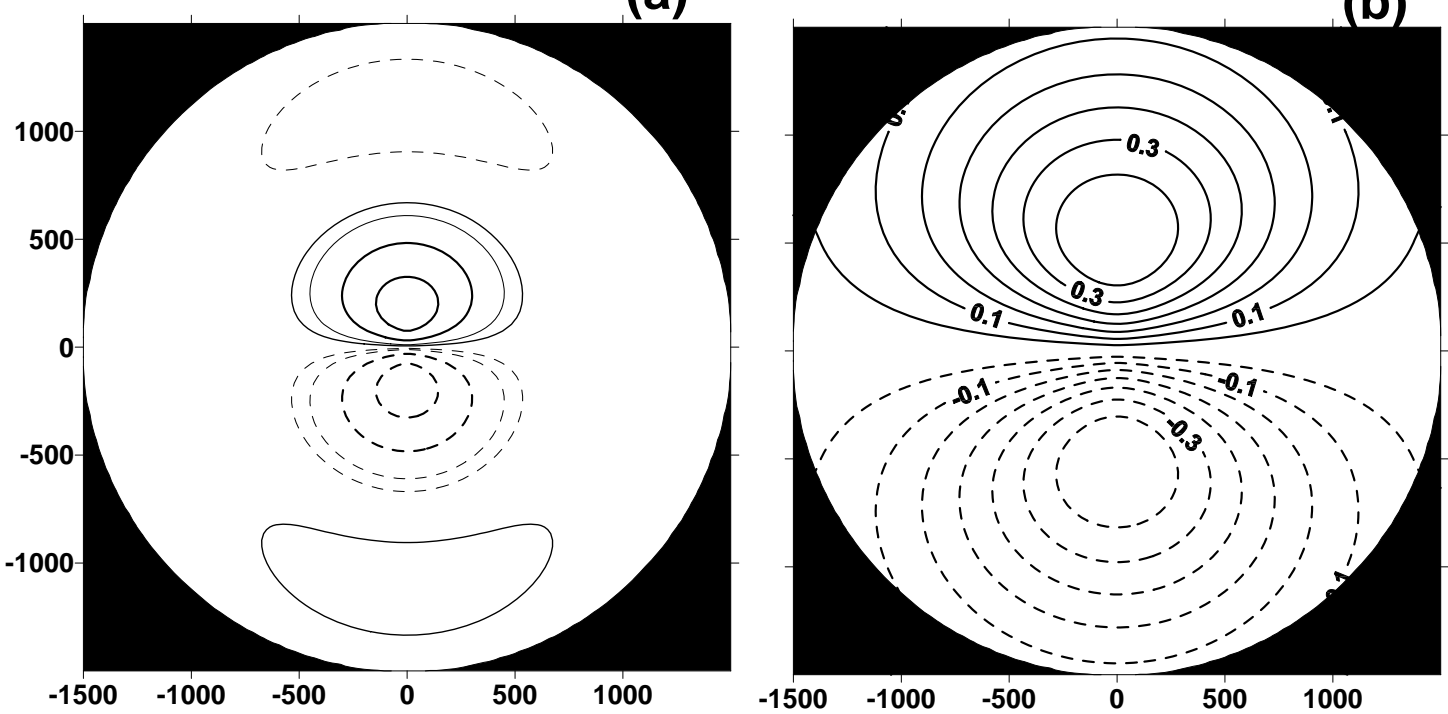

Figure 3. Contour plot of the wind stress curl (21) when (a) $\alpha=6 / a$; (b) $\alpha=2 / a$. Contour interval is 0.05 .

which decays to zero for large $r$. We find that

$$
\boldsymbol{k} \cdot \boldsymbol{\nabla} \times\left(\frac{\boldsymbol{\tau}}{\rho H}\right)=\mathrm{F}=\frac{1}{r} \frac{\partial}{\partial r}\left(\frac{r \tau^{\phi}}{\rho H}\right)=\frac{\tau_{0} \sin \phi}{\rho H a^{2}}(3-\alpha r) r \mathrm{e}^{-\alpha r},
$$

and from (5) we observe that the velocity field is bounded at the origin. Figure 3 shows contours of (21) for two values of the parameter $\alpha$. In figure 3(a) the wind stress curl vanishes at $r=a / 2$ and we refer to this type of field as a mode 2 radial structure. In contrast, the curl in figure 3(b) has a mode 1 radial structure. The interior Sverdrup balance is given by

$$
\frac{f}{R^{2}} \frac{\partial \Psi_{I}}{\partial \phi}=\mathrm{F}
$$

which upon integration yields

$$
\Psi_{I}=-\frac{\tau_{0} R^{2}}{\rho f H a^{2}} \cos \phi(3-\alpha r) r \mathrm{e}^{-\alpha r} .
$$

Using the boundary layer analysis above we find that the total solution is given by

$$
\begin{aligned}
\Psi= & -\frac{\tau_{0} R^{2}}{\rho f H a^{2}} \cos \phi(3-\alpha r) r \mathrm{e}^{-\alpha r} \\
& +\frac{\tau_{0} R^{2}}{\rho f H a} \cos \phi(3-\alpha a) \mathrm{e}^{-\alpha a} \exp \left[-\frac{s}{\sqrt{2} R}\right] \cos \left[\frac{s}{\sqrt{2} R}+\phi\right] .
\end{aligned}
$$

Figure 4 shows contours of the non-dimensional form of (23) when $\alpha=6 / a$ (in which case 

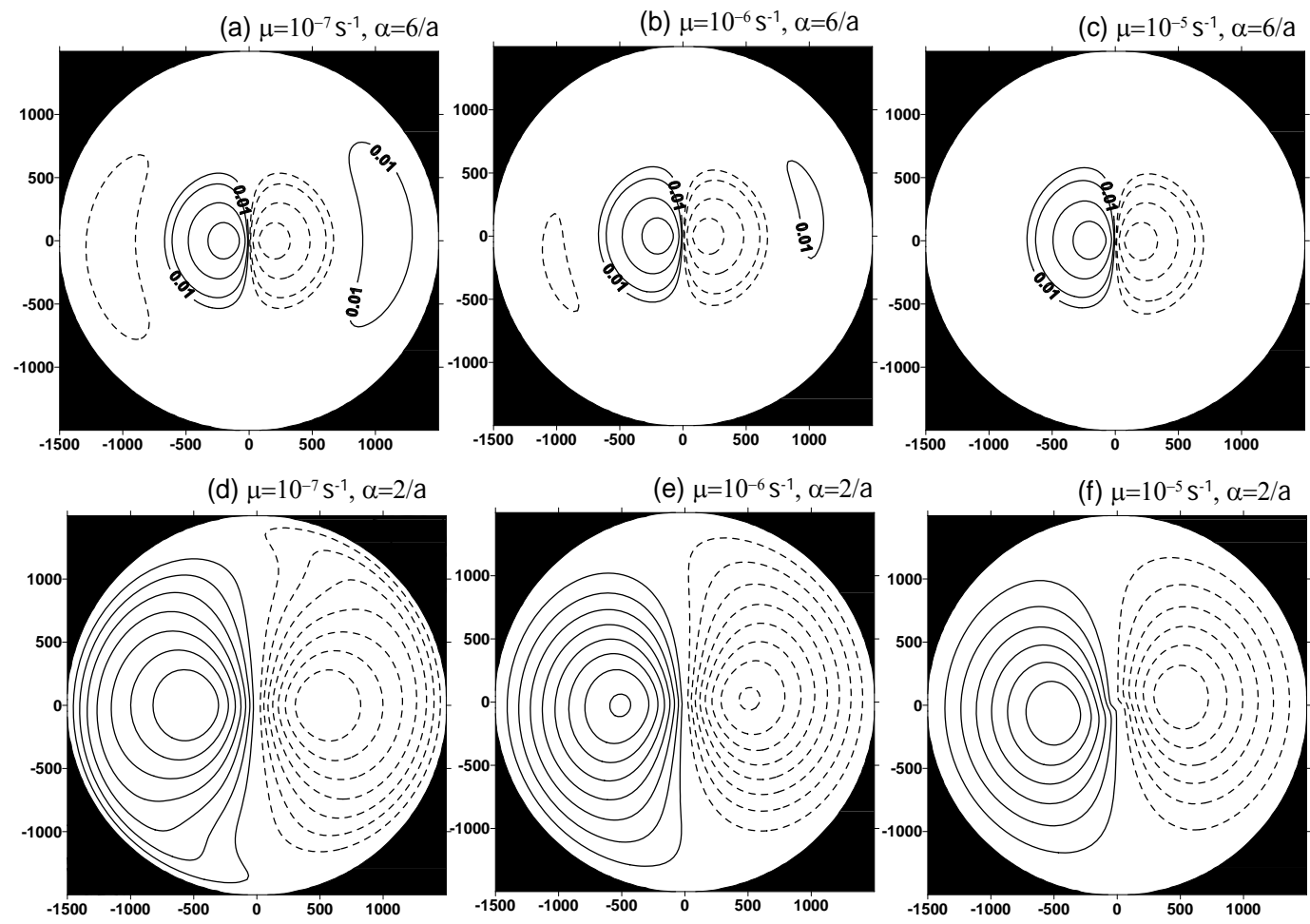

Figure 4. Contour plots of the dimensionless velocity streamfunction (23). Panels (a-c) correspond to $\mu=10^{-7} \mathrm{~s}^{-1}$, $10^{-6} \mathrm{~s}^{-1}$ and $10^{-5} \mathrm{~s}^{-1}$, respectively, where $\alpha^{-1}$, the e-folding radial decay scale of the wind stress curl (21) is given by $\alpha^{-1}=a / 6$. Panels (d-f) are analogous to except $\alpha^{-1}=a / 2$. Contour interval is 0.05 .

$\mathrm{F}=0$ at $r=a / 2$ ) and when $\alpha=2 / a$. Equation (23) is non-dimensionalised using $\Psi_{0}$ as defined in the dimensionless form of (20). As noted in figure 2, the streamline pattern rotates counter clockwise with increasing bottom friction. For the radial mode 2 solution shown in figure 4 (ac) the cells adjacent to the basin wall are all but removed by friction when $\mu=10^{-5} \mathrm{~s}^{-1}$. In contrast, the two cell flow that is confined to the basin interior in figure $4(\mathrm{~d}-\mathrm{f})$ preserves its structure as bottom friction increases and exhibits only slight counter clockwise rotation.

\section{Boundary forced solutions}

The Arctic basin is connected to the North Atlantic via the Greenland-Iceland-Norwegian (GIN) Sea and the Labrador Sea and to the North Pacific via the Bering Strait. Viewing a prototype model of the Arctic Ocean as a circular basin, the exchange of water masses between this basin and the North Atlantic and North Pacific is represented by a prescribed streamfunction at the boundary $r=a$. This approach is well illustrated by representing the exchange of water masses through the GIN Sea driving a circulation in the Arctic basin. For convenience we will neglect wind stress forcing in this section and we consider the solution of the boundary forced problem

$$
\mu r \nabla^{2} \Psi+\frac{r f}{R^{2}} \frac{\partial \Psi}{\partial \phi}=0
$$


subject to

$$
\Psi=\Psi_{B}(\phi) \quad \text { on } \quad r=a
$$

Specifically we consider

$$
\Psi_{B}(\phi)= \begin{cases}\frac{T}{2 H} \cos \left(\frac{\pi \phi}{\widehat{\phi}}\right), & -\widehat{\phi} \leq \phi \leq \widehat{\phi} \\ 0, & \text { otherwise }\end{cases}
$$

where $T$ is the volume transport across $[0, \widehat{\phi}], H$ is the depth of the ocean and arc length $2 a \widehat{\phi}$ is representative of the width of the GIN Sea at $70 \mathrm{~N}^{\circ}$, say. Across the open boundary we see from (26) that

$$
a u=-\frac{\partial \Psi_{B}}{\partial \phi}=\frac{\pi T}{2 H \widehat{\phi}} \sin \left(\frac{\pi \phi}{\widehat{\phi}}\right)
$$

and therefore the exchange of water across the open boundary, $r=a, 0 \leq \phi \leq \widehat{\phi}$ is given by

$$
V=\int_{0}^{\widehat{\phi}} a u H \mathrm{~d} \phi=\frac{\pi T}{2 \widehat{\phi}} \int_{0}^{\widehat{\phi}} \sin \left(\frac{\pi \phi}{\widehat{\phi}}\right) \mathrm{d} \phi=T,
$$

using (27), with an equal and opposite volume transport across $-\widehat{\phi} \leq \phi \leq 0$.

\subsection{Exact solution}

We seek a $2 \pi$-periodic solution of (24) of the form

$$
\widehat{\Psi}=\sum_{n=-\infty}^{\infty} G_{n}(r) \mathrm{e}^{\mathrm{i} n \phi} .
$$

Hereafter, we treat $f$ as constant in (24). Substituting (28) into (24) yields the governing equation for $G_{n}$ :

$$
r^{2} \frac{\mathrm{d}^{2} G_{n}}{\mathrm{~d} r^{2}}+r \frac{\mathrm{d} G_{n}}{\mathrm{~d} r}+\left(-n^{2}+\frac{\mathrm{i} n f r^{2}}{\mu R^{2}}\right) G_{n}=0
$$

The bounded solution of (29) is given by

$$
G_{n}=a_{n} \mathrm{~J}_{n}\left(\frac{r}{R}\left(\frac{\mathrm{i} n f}{\mu}\right)^{1 / 2}\right)
$$

where $\mathrm{J}_{n}$ is the Bessel function of the first kind of order $n$ and $a_{n}$ is an arbitrary constant. Applying the boundary condition (26) and using (28) and (30) we obtain

$$
\sum_{n=-\infty}^{\infty} a_{n} \mathrm{~J}_{n}\left(\frac{a}{R}\left(\frac{\mathrm{i} n f}{\mu}\right)^{1 / 2}\right) \mathrm{e}^{\mathrm{i} n \phi}=\left\{\begin{array}{lc}
\frac{T}{2 H} \cos \left(\frac{\pi \phi}{\widehat{\phi}}\right), & -\widehat{\phi} \leq \phi \leq \widehat{\phi} \\
0, & \text { otherwise }
\end{array}\right.
$$

which enables the coefficients $a_{n}$ to be determined:

$$
a_{n} \mathrm{~J}_{n}\left(\frac{a}{R}\left(\frac{\mathrm{i} n f}{\mu}\right)^{1 / 2}\right) \mathrm{e}^{\mathrm{i} n \phi}=\frac{1}{2 \pi} \int_{-\widehat{\phi}}^{\widehat{\phi}} \frac{T}{2 H} \cos \left(\frac{\pi \phi}{\widehat{\phi}}\right) \mathrm{e}^{-\mathrm{i} n \phi} \mathrm{d} \phi .
$$


We find that

$$
a_{n}=\frac{T n \sin (n \widehat{\phi})}{2 H \pi \mathrm{DJ}_{n}\left(a(\mathrm{i} n f)^{1 / 2} / R \sqrt{\mu}\right)}, \quad \forall n, \quad \text { where } \quad \mathrm{D} \equiv n^{2}-\left(\frac{\pi}{\widehat{\phi}}\right)^{2} .
$$

Finally, after some algebra the exact solution can be written

$$
\Psi=\frac{T}{\pi H} \operatorname{Re}\left\{\sum_{n=1}^{\infty} \frac{n \sin (n \widehat{\phi})}{\mathrm{D}} \frac{\mathrm{J}_{n}\left(r(\mathrm{i} n f)^{1 / 2} / R \sqrt{\mu}\right)}{\mathrm{J}_{n}\left(a(\mathrm{i} n f)^{1 / 2} / R \sqrt{\mu}\right)} \mathrm{e}^{\mathrm{i} n \phi}\right\}
$$

where Re denotes the real part of the complex function. Computationally, evaluation of (33) requires care due to the complex arguments of the Bessel functions. Therefore, we enquire whether a more computationally straightforward, albeit approximate solution, can be obtained.

\subsection{Approximate solution}

Recall that the frictional boundary layer solution takes the form

$$
\begin{aligned}
\widehat{\Psi}= & \sum_{n=-\infty}^{0} a_{n} \exp \left[-(1+\mathrm{i}) \frac{s}{R} \sqrt{\frac{|n|}{2}}\right] \exp (\mathrm{i} n \phi) \\
& +\sum_{n=1}^{\infty} a_{n} \exp \left[-(1-\mathrm{i}) \frac{s}{R} \sqrt{\frac{n}{2}}\right] \exp (\mathrm{i} n \phi), \quad \text { where } \quad s=\frac{a-r}{\sqrt{\mu / f}} .
\end{aligned}
$$

On $s=0$ (i.e., $r=a$ ) we require that

$$
\widehat{\Psi}(0, \phi)=\sum_{n=-\infty}^{\infty} a_{n} \mathrm{e}^{\mathrm{i} n \phi}=\left\{\begin{array}{lc}
\frac{T}{2 H} \cos \left(\frac{\pi \phi}{\widehat{\phi}}\right), & -\widehat{\phi} \leq \phi \leq \widehat{\phi} \\
0, & \text { otherwise }
\end{array}\right.
$$

We find that

$$
a_{n}=\frac{T n \sin (n \widehat{\phi})}{2 \pi H \mathrm{D}}
$$

where $\mathrm{D}$ is defined by (32b). Finally, the approximate boundary forced solution takes the form

$$
\widehat{\Psi}=-\frac{T}{\pi H} \sum_{n=1}^{\infty} \frac{\sin (n \widehat{\phi})}{\mathrm{D}} \exp \left[-\frac{s}{R} \sqrt{\frac{n}{2}}\right] \cos \left(\frac{s}{R} \sqrt{\frac{n}{2}}+n \phi\right) .
$$

Figures 5 and 6 show contour plots of the non-dimensional forms of (33) and (34), respectively, for a range of values of $\mu$. The scaling factor used in the non-dimensionalisation is $\Psi_{0}=T /(2 H)$. Other parameter values are listed in Table 1 . When bottom friction is small the recirculating cells are oriented in the direction of the phase velocity of planetary Rossby waves (clockwise) that would be responsible for establishing the steady flow in a spin-up problem form rest. As bottom friction increases the cells become less deflected and are becomes aligned in a radial direction, albeit with decreased magnitude. Figure 6 shows that although the approximate solution qualitatively reproduces the flow characteristics it overestimates the radial penetration of the flow into the basin. 
(a) $\mu=10^{-7} \mathrm{~s}^{-1}$

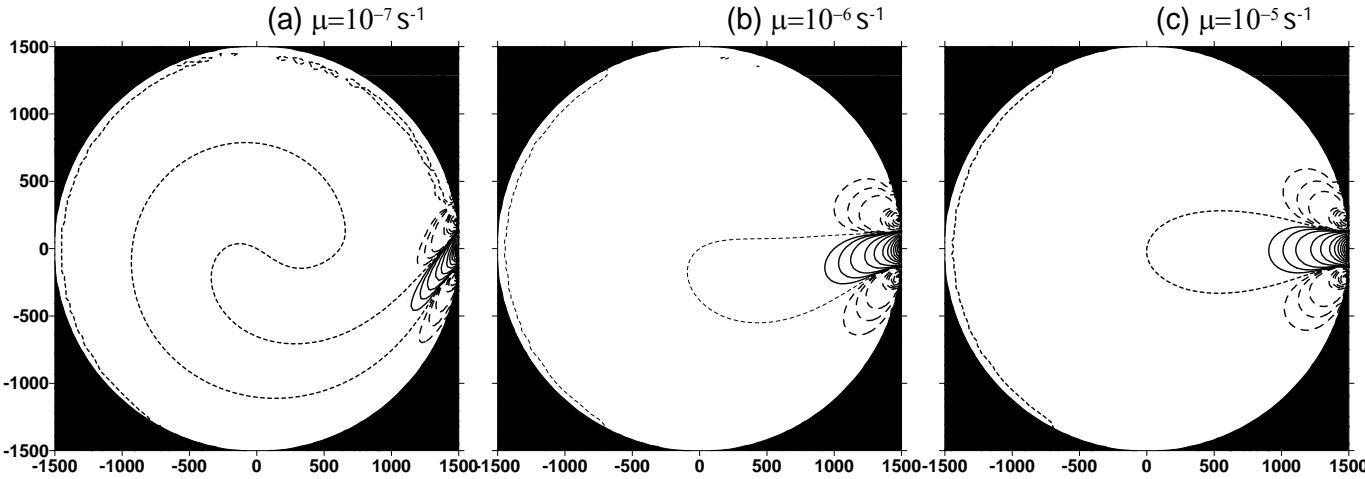

Figure 5. Contour plots of the dimensionless velocity streamfunction (33) driven by inflow and outflow across $[-\widehat{\phi}, \widehat{\phi}]$ when; (a) $\mu=10^{-7} \mathrm{~s}^{-1}$; (b) $\mu=10^{-6} \mathrm{~s}^{-1}$; (c) $\mu=10^{-5} \mathrm{~s}^{-1}$. Contour values are $\pm 0.01, \pm 0.02, \pm 0.05, \pm 0.1, \pm 0.2$, $\cdots, \pm 0.8$.

\section{Boundary forced circulation in a basin with a shelf}

We extend the boundary forced solutions discussed in section 4 by introducing a step shelf of uniform depth $H_{1}$ and uniform width $(1-\varepsilon) a$, where $0 \leq \varepsilon \leq 1$. A schematic of the basin with the step shelf is shown in figure 7. Region 1 refers to the shelf and Region 2 refers to the deep inner circular basin of radius $\varepsilon a$ and depth $\mathrm{H}_{2}$. For a non-uniform depth basin it is convenient to define a volume transport streamfunction, rather than the velocity streamfunction used above, because the matching conditions at the shelf edge $r=\varepsilon a$ are simplified. The volume transport stream function $\Psi$ is defined as

$$
H r u=-\frac{\partial \Phi}{\partial \phi}, \quad \frac{H v}{\cos \theta}=\frac{\partial \Phi}{\partial r},
$$

which leads to a minor modification of the prescribed transport inflow/outflow boundary condition (26), namely

$$
\Phi_{B}(\phi)=\left\{\begin{array}{lr}
\frac{T}{2} \cos \left(\frac{\pi \phi}{\widehat{\phi}}\right), & -\widehat{\phi} \leq \phi \leq \widehat{\phi}, \\
0, & \text { otherwise. }
\end{array}\right.
$$

The governing equation for $\Phi$ is again given by (24) subject to the boundary condition (25), where $\Phi_{B}$ is now given by (36). Following the identical method of solution used in section 4 
(a) $\mu=10^{-7} \mathrm{~s}^{-1}$

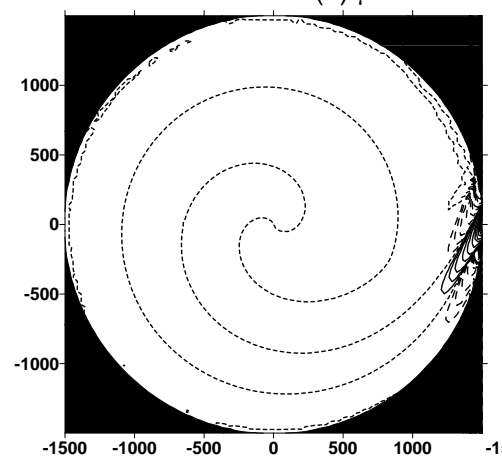

(b) $\mu=10^{-6} \mathrm{~s}^{-1}$

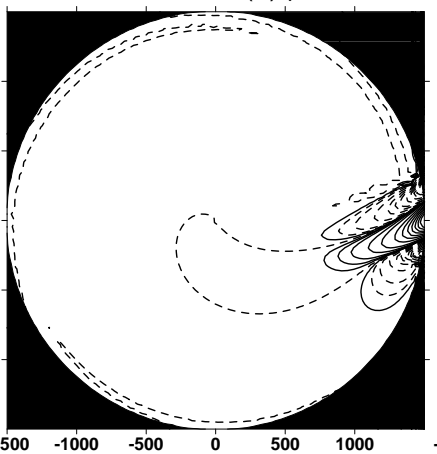

(c) $\mu=10^{-5} \mathrm{~S}^{-1}$

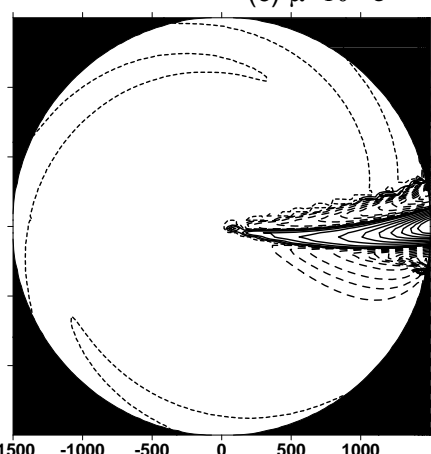

Figure 6. As in figure 5, except that the approximate boundary layer solution (34) is contoured.

we find that the solutions for $\Phi_{j}$ in Region $j(j=1,2)$ are given by

$$
\begin{aligned}
& \Phi_{1}=\sum_{n=-\infty}^{\infty}\left[b_{n} \mathrm{~J}_{n}\left(\frac{r}{R}\left(\frac{\mathrm{i} n f}{\mu}\right)^{1 / 2}\right)+c_{n} \mathrm{Y}_{n}\left(\frac{r}{R}\left(\frac{\mathrm{i} n f}{\mu}\right)^{1 / 2}\right)\right] \mathrm{e}^{\mathrm{i} n \phi}, \\
& \Phi_{2}=\sum_{n=-\infty}^{\infty} a_{n} \mathrm{~J}_{n}\left(\frac{r}{R}\left(\frac{\mathrm{i} n f}{\mu}\right)^{1 / 2}\right) \mathrm{e}^{\mathrm{i} n \phi},
\end{aligned}
$$

where the coefficients $a_{n}, b_{n}$ and $c_{n}$ are to be determined and $\mathrm{Y}_{n}$ denotes the Bessel function of order $n$ of the second kind.

At the shelf edge continuity of the normal volume transport and pressure yield, respectively, the following matching conditions:

$$
\begin{aligned}
& {[\Phi]=0, \quad \text { at } \quad r=\varepsilon a,} \\
& {\left[\frac{f}{r H} \frac{\partial \Phi}{\partial \phi}+\frac{\mu}{H} \frac{\partial \Phi}{\partial r}\right]=0, \quad \text { at } \quad r=\varepsilon a \text {. }}
\end{aligned}
$$

Applying (36) we obtain

$$
b_{n} \mathrm{~J}_{n}\left(\frac{a}{R}\left(\frac{\mathrm{i} n f}{\mu}\right)^{1 / 2}\right)+c_{n} \mathrm{Y}_{n}\left(\frac{a}{R}\left(\frac{\mathrm{i} n f}{\mu}\right)^{1 / 2}\right)=-\frac{T}{2 \pi} \frac{n \sin (n \widehat{\phi})}{\mathrm{D}},
$$




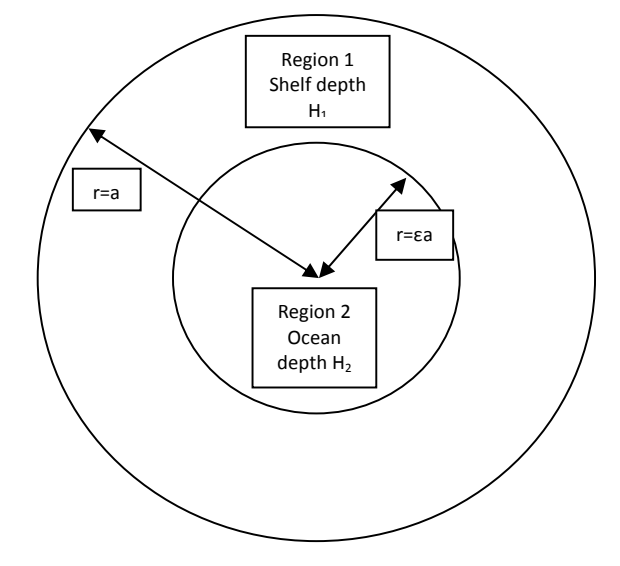

\author{
-
}

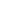


while application of the matching conditions (38) yields

$$
a_{n} \mathrm{~J}_{n}\left(\frac{\varepsilon a}{R}\left(\frac{\mathrm{i} n f}{\mu}\right)^{1 / 2}\right)=b_{n} \mathrm{~J}_{n}\left(\frac{\varepsilon a}{R}\left(\frac{\mathrm{i} n f}{\mu}\right)^{1 / 2}\right)+c_{n} \mathrm{Y}_{n}\left(\frac{\varepsilon a}{R}\left(\frac{\mathrm{i} n f}{\mu}\right)^{1 / 2}\right)
$$

and

$$
\begin{gathered}
\frac{(\mu f)^{1 / 2}}{2 H_{1} R}\left\{b_{n}\left[\mathrm{~J}_{n-1}\left(\frac{\varepsilon a}{R}\left(\frac{\mathrm{i} n f}{\mu}\right)^{1 / 2}\right)-\mathrm{J}_{n+1}\left(\frac{\varepsilon a}{R}\left(\frac{\mathrm{i} n f}{\mu}\right)^{1 / 2}\right)\right]\right. \\
\left.+c_{n}\left[\mathrm{Y}_{n-1}\left(\frac{\varepsilon a}{R}\left(\frac{\mathrm{i} n f}{\mu}\right)^{1 / 2}\right)-\mathrm{Y}_{n+1}\left(\frac{\varepsilon a}{R}\left(\frac{\mathrm{i} n f}{\mu}\right)^{1 / 2}\right)\right]\right\} \\
=a_{n}\left\{\frac{(\mu f)^{1 / 2}}{2 H_{2} R}\left[\mathrm{~J}_{n-1}\left(\frac{\varepsilon a}{R}\left(\frac{\mathrm{i} n f}{\mu}\right)^{1 / 2}\right)-\mathrm{J}_{n+1}\left(\frac{\varepsilon a}{R}\left(\frac{\mathrm{i} n f}{\mu}\right)^{1 / 2}\right)\right]\right. \\
\left.-\frac{f(\Delta H)(\mathrm{i} n)^{1 / 2}}{\varepsilon a H_{1} H_{2}} \mathrm{~J}_{n}\left(\frac{\varepsilon a}{R}\left(\frac{\mathrm{i} n f}{\mu}\right)^{1 / 2}\right)\right\} .
\end{gathered}
$$

In (41), we have $\Delta H=H_{2}-H_{1}>0$. Inspection of (39) to (41) revealed that $a_{0}=b_{0}=c_{0}=0$ and that

$$
e_{-2 m}=e_{2 m}^{*}, \quad e_{-(2 m-1)}=-e_{2 m-1}^{*}
$$

where $e_{m}$ is any one of the family of coefficients $a_{m}, b_{m}$, and $c_{m}(m=1,2,3, \cdots)$, and the asterisk denotes complex conjugate. Using (42) we can simplify the solutions (37):

$$
\begin{aligned}
& \Phi_{1}=2 \operatorname{Re}\left\{\sum_{n=1}^{\infty}\left[b_{n} \mathrm{~J}_{n}\left(\frac{r}{R}\left(\frac{\mathrm{i} n f}{\mu}\right)^{1 / 2}\right)+c_{n} \mathrm{Y}_{n}\left(\frac{r}{R}\left(\frac{\mathrm{i} n f}{\mu}\right)^{1 / 2}\right)\right] \mathrm{e}^{i n \phi}\right\}, \\
& \Phi_{2}=2 \operatorname{Re}\left\{\sum_{n=1}^{\infty} a_{n} \mathrm{~J}_{n}\left(\frac{r}{R}\left(\frac{\mathrm{i} n f}{\mu}\right)^{1 / 2}\right) \mathrm{e}^{i n \phi}\right\} .
\end{aligned}
$$

Contour plots of the solutions (43), non-dimensionalised by $T / 2$, are plotted for a range of values of bottom friction and shelf width and depth. The step shelf in figure 8 is relatively shallow compared with the depth of the deep basin with $H_{1}=500 \mathrm{~m}$ and $H_{2}=4000 \mathrm{~m}$. In figures 8 (a-c) the bottom friction coefficient $\mu=10^{-6} \mathrm{~s}^{-1}$ and we observe that the flow is confined to the shelf in all cases, with the axes of the circulation cells oriented in the direction of the phase velocity of planetary waves. When $\mu=10^{-5} \mathrm{~s}^{-1}$ in figure $8(\mathrm{~d}-\mathrm{f})$ the flow is again confined to the shelf with the axes of the circulation cells oriented zonally. Figure 9 shows the effect of decreasing the depth of the deep basin to $1000 \mathrm{~m}$. In figure 9 (a-c) $\mu=10^{-5} \mathrm{~s}^{-1}$ and we observe that significant flow occurs in the deep basin, especially as the shelf width decreases. Frictional boundary layers centred at the shelf edge are responsible for breaking the severe constraint that steady geostrophic flow is aligned along isobaths and allow fluid to cross the shelf break. Indeed, when bottom friction is reduced $\left(\mu=10^{-6} \mathrm{~s}^{-1}\right)$ in figure $9(\mathrm{~d})$ compared with figure $9(\mathrm{c})$ we see that the flow is once again confined to the shelf.

In summary, when bottom friction is of the order of $10^{-5} \mathrm{~s}^{-1}$ and for small $\Delta H$ we find that significant flow crosses the shelf break.

\section{Discussion}

It is self-evident that in a closed polar basin, of the type considered in this study, that the circulation will be characterised by gyres. We observe significant differences in the gyre struc- 
(a) $\varepsilon=0.5, \mu=10^{-6} \mathrm{~s}^{-1}$

(b) $\varepsilon=0.7, \mu=10^{-6} \mathrm{~S}^{-1}$

(c) $\varepsilon=0.8, \mu=10^{-6} \mathrm{~s}^{-1}$
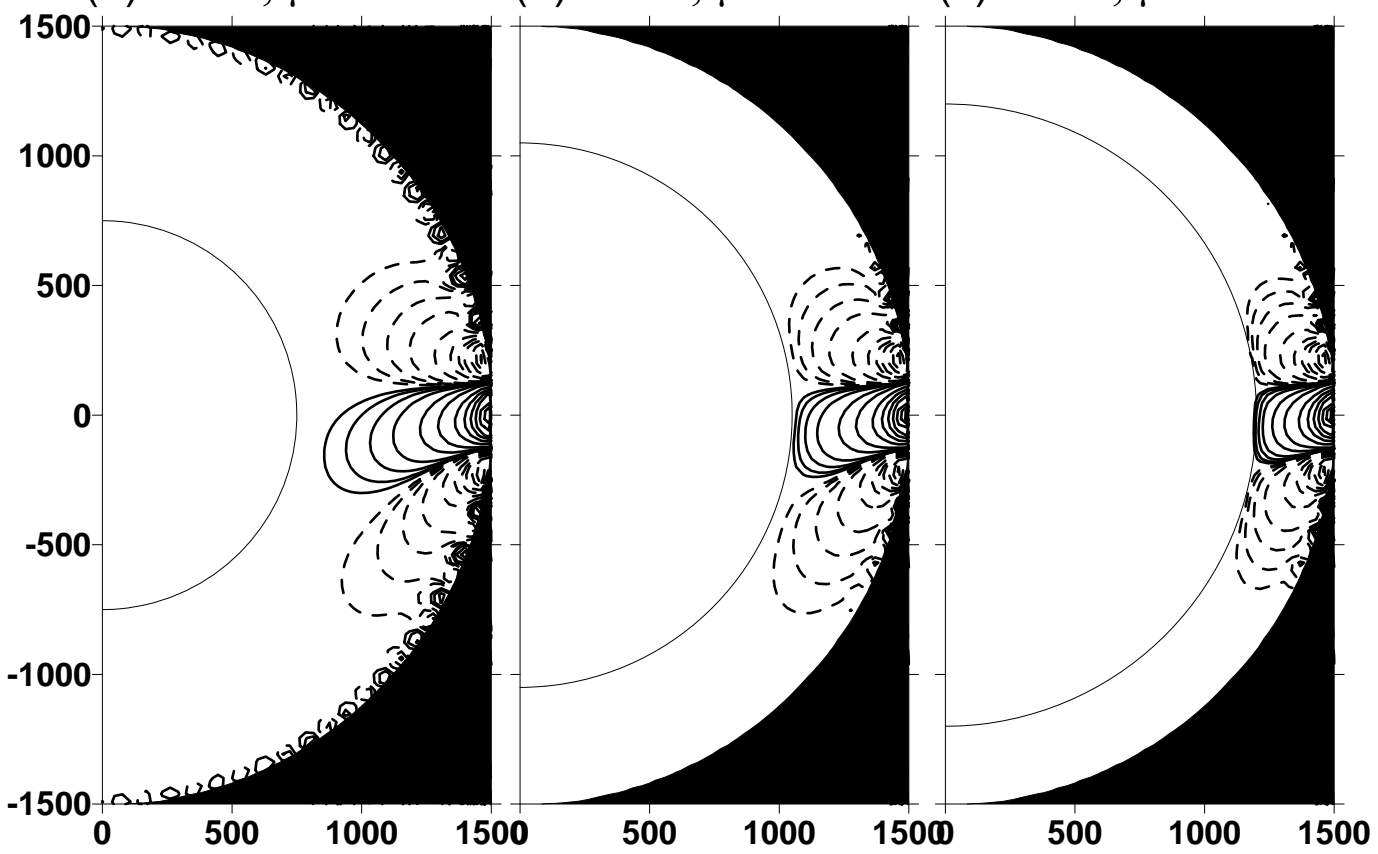

(d) $\varepsilon=0.5, \mu=10^{-5} \mathrm{~s}^{-1}$

(e) $\varepsilon=0.7, \mu=10^{-5} \mathrm{~s}^{-1}$

(f) $\varepsilon=0.8, \mu=10^{-5} \mathrm{~s}^{-1}$
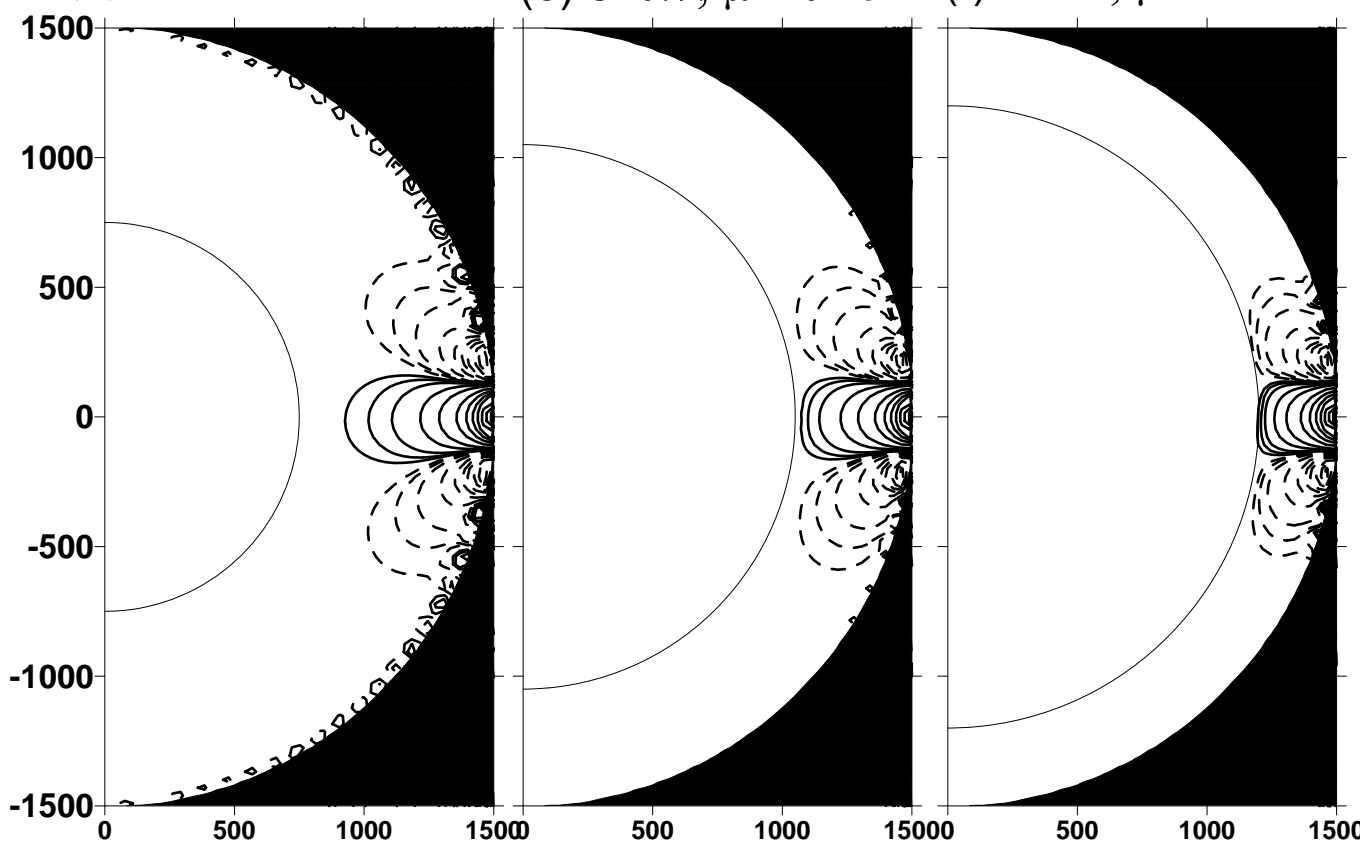

Figure 8. Contour plot of the dimensionless transport streamfunction (43), $H_{1}=500 \mathrm{~m}, H_{2}=4000 \mathrm{~m}$. The shelf width is $(1-\varepsilon a)$, where $\varepsilon=0.5,0.7$ and 0.8 in (a-c), respectively, and $\mu=10^{-6} \mathrm{~s}^{-1}$ is fixed. Contour plots (d-f) as in (a-c), except that $\mu=10^{-5} \mathrm{~s}^{-1}$. Semi-circular contours designate the locations of shelf. Contour values are $\pm 0.0025, \pm 0.005$, $\pm 0.01, \pm 0.02, \pm 0.05, \pm 0.06, \pm 0.07, \pm 0.08$. 


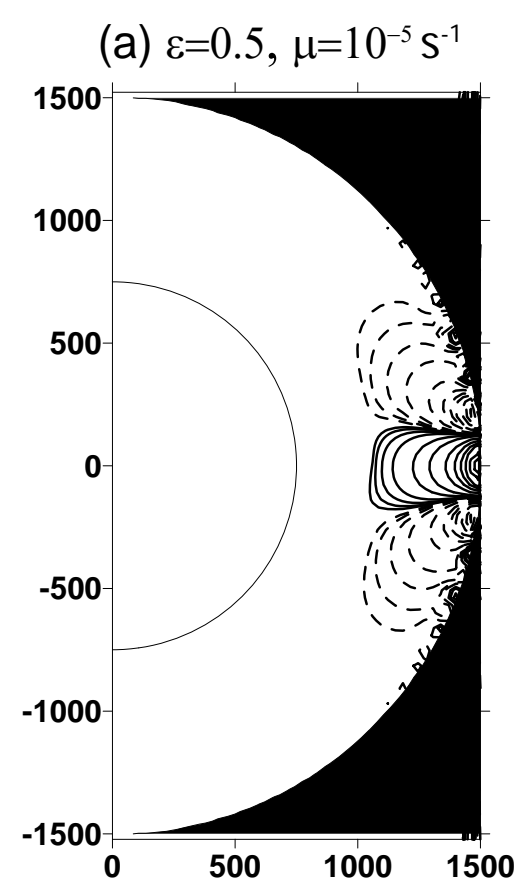

(b) $\varepsilon=0.7, \mu=10^{-5} \mathrm{~s}^{-1}$
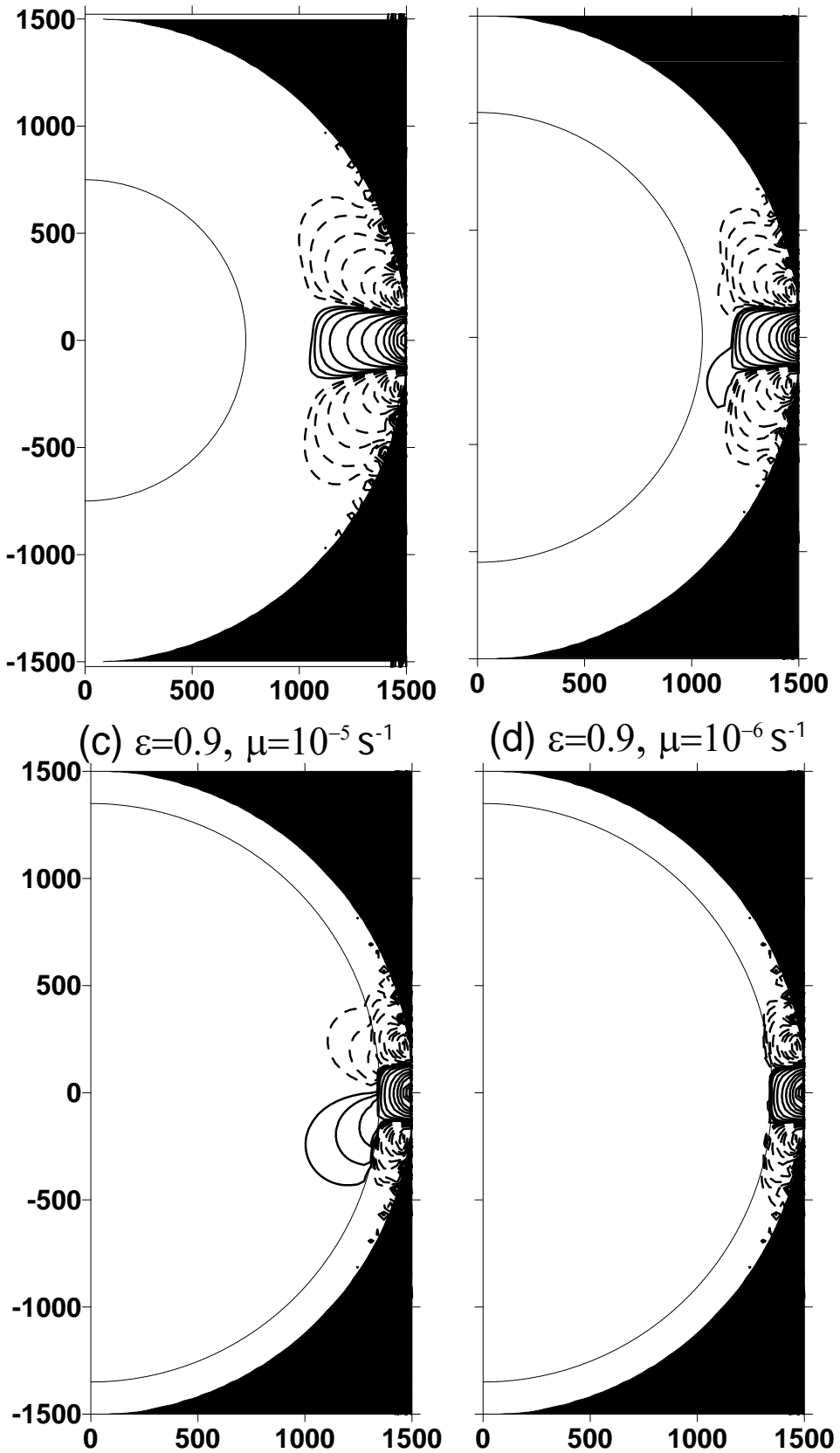

(d) $\varepsilon=0.9, \mu=10^{-6} \mathrm{~s}^{-1}$

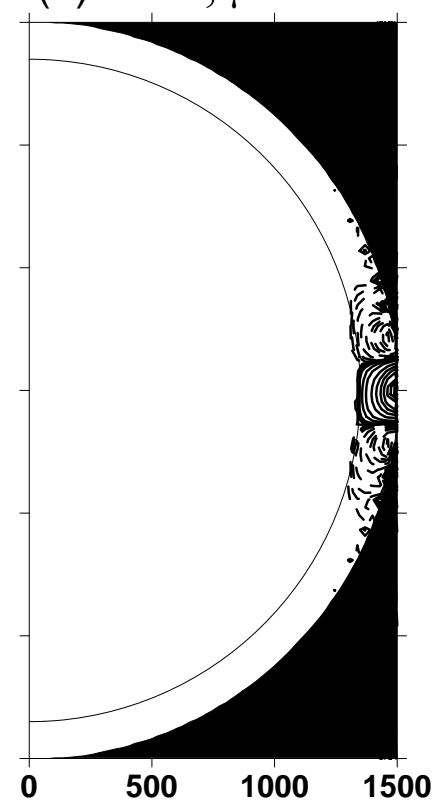

Figure 9. As in figure 8, except $H_{1}=500 \mathrm{~m}, H_{2}=1000 \mathrm{~m}$ and $\varepsilon=0.5,0.7$ and 0.9 in (a-c) respectively, with $\mu=10^{-5} \mathrm{~s}^{-1}$ fixed. In plot (d) $\mu=10^{-6} \mathrm{~s}^{-1}$ and $\varepsilon=0.9$. Contour values are $\pm 0.0025, \pm 0.005, \pm 0.01, \pm 0.02, \pm 0.05$ \pm 0.08 . 
ture in a basin on an $f$-plane compared with the equivalent basin on a polar plane (e.g., comparing figures A1 and 2(a)) despite the fact that the changes in the value of the Coriolis parameter with distance from the pole are small. In practice, the gyre structure is controlled by the external forcing (i.e., wind stress, buoyancy forcing and cross-basin pressure gradients associated with water mass exchange with the marginal seas), stratification, topography and dissipative processes. Sea ice can also significantly modify the shear stress at the top of the water column wherever the ice is not in free-drift. Clearly, incorporation of all these factors in one of the first analytical models of the steady-state circulation in a closed circular basin on a polar plane would be unrealistic. Instead, the approach adopted in this study is to develop new solutions for the steady-state wind and boundary driven circulation in closed circular basin located on a polar plane. Only the simplest topography is considered, namely a step shelf of uniform width. The effects of stratification, major ridges, and time dependent dynamical features such as eddies and sea ice are neglected. Nevertheless, the value of this model is that it provides an independent method of assessing the fidelity of ocean circulation models of the Arctic Ocean.

Although the simplest models of the Arctic Ocean treat the basin as a closed circular basin centred at the pole the reality is very different. The Bering Strait allows exchange of water masses between the Arctic and the Pacific. Similarly the Canadian Archipelago and the Greenland Iceland-Norwegian Seas allow exchange of water masses between the Arctic and the Atlantic basins. These water mass exchanges play a key role in the formation of dense and intermediate water masses (Woodgate et al. 2005, Schauer et al. 2002). In an extension to the work presented here we suggest that it would be appropriate to study the steady state circulation in a polar basin driven by sources and sinks of water located on the boundary of the basin. Additionally, a first attempt at analytically modelling the important effects of stratification would be to use a 2-layer model to capture the pronounced stratification observed in the upper $150 \mathrm{~m}$ of the water column (Woodgate 2005).

\section{Acknowledgements}

We thank the referees for their constructive reviews of the manuscript which have improved its presentation. MVL thanks the Forum for Arctic Ocean Modeling and Observational Synthesis (FAMOS) for stimulating new ideas and constructive discussions about the results reported in this paper.

\section{References}

Harlander, U., A high-latitude quasi-geostrophic delta plane model derived from spherical geometry. Tellus $2005, \mathbf{5 7}, 43-54$.

Imawaki, S. and Takano, K., Planetery flow in a circular basin. Deep-Sea Res. 1974, 21, 69-77.

LeBlond, P.H., Planetary waves in a symmetrical polar basin. Tellus 1964, 16, 503-511.

Luneva, M., Willmott, A.J. and Morales Maqueda, M.A., Geostrophic adjustment problems in a polar basin. Atmosphere-Ocean 2012, 50, 134-155.

Nøst, O.A. and Isachsen, P.E., The large-scale time-mean ocean circulation in the Nordic Seas and Arctic Ocean estimated from simplied dynamics. J. Mar. Res. 2003, 61, 175-210.

Proshutinsky, A.Y. and Johnson, M.A., Two circulation regimes of the wind-driven Arctic Ocean. J. Geophys. Res. 1997, 102, 12,493-12,514.

Proshutinsky, A.Y. and Johnson, M.A., Two regimes of the Arctic's circulation from ocean models with ice and contaminants. Mar. Pollution Bull. 2001, 43, 61-70.

Proshutinsky, A.Y., Frishfield, R., Timmermans, M.L., Toole, J., Carmack, E., McLaughlin, F., Williams, W.J., Zimmermann, S., Itoh, M. and Shimada, K., Beaufort Gyre freshwater reservoir: State and variability from observations. J. Geophys. Res. 2009, 114, C00A10, 1-25, doi: 10.1029/2008JC005104.

Schauer, U., Loeng, H., Rudels, B., Ozhigin, V.K. and Dieck, W., Atlantic Water flow through the Barents and Kara Seas. Deep-Sea Res. 2002, 49, 2281-2298. 
Wang, M. and Overland, J.E., A sea ice free summer Arctic within 30 years? Geophys. Res. Lett. 2009, 36, L07502, 1-5, doi: 10.1029/2009GL037820.

Woodgate, R.A., Aagaard, K. and Weingartner, T.J., Monthly temperature, salinity, and transport variability of the Bering Strait. Geophys. Res. Lett. 2005, 32, L04601 1-4, doi: 10.1029/2004GL021880.

Woodgate, R.A., Arctic Ocean circulation: going around at the top of the world. Nature Education Knowledge $2013,4,8$.

\section{Appendix A: $f$-plane solutions}

Neglecting variations of $f$ with latitude, the vorticity equation (8) reduces to Poisson's equation

$$
\mu \nabla^{2} \Psi=r \boldsymbol{k} \cdot \nabla \times\left(\frac{\boldsymbol{\tau}}{\rho H}\right)
$$

It is straightforward to obtain the solution of (A.1) for wind stress curl (14):

$$
\Psi=\frac{\tau_{0} r\left(r^{2}-a^{2}\right) \sin \phi}{8 \rho H a^{2} \mu} .
$$

A contour plot of (A.2), normalised by the factor $a \tau_{0} /(8 \rho H \mu)$, is plotted in figure A1. On the $f$-plane the wind driven solution corresponds to a pair of anti-symmetric gyres with the flow in geostrophic balance.

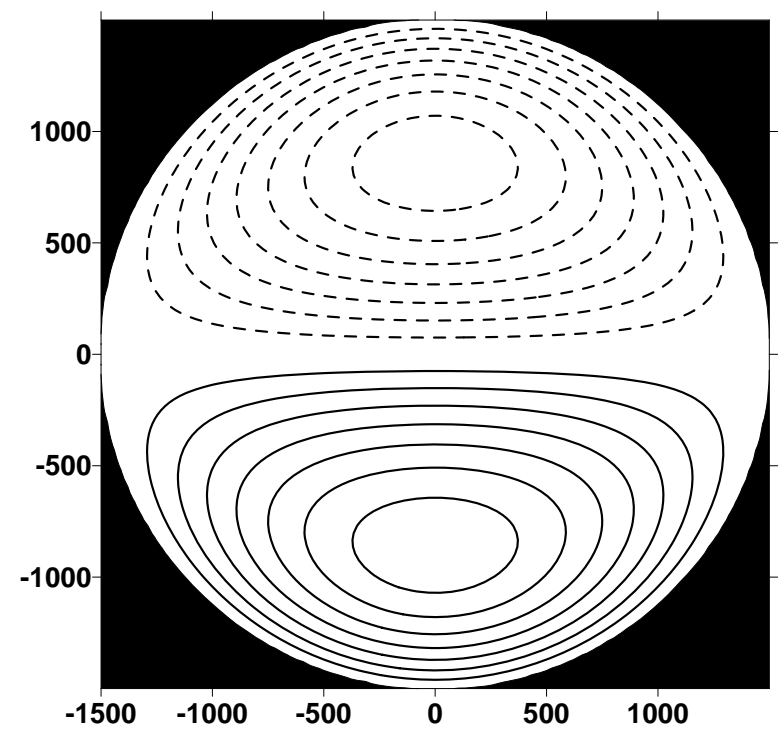

Figure A1. Contour plot of the dimensionless velocity streamfunction (A.2) on an $f$-plane for comparison with the polar-plane plot in figure $2(\mathrm{a})$.

The equivalent $f$-plane solution for wind stress curl (21) is given by

$$
\Psi=\frac{\tau_{0} \sin \phi}{20 \rho H a^{2} \mu} r \mathrm{e}^{-a r}\left[r^{2}-13 \frac{r}{\alpha}-\frac{13}{\alpha^{2}}\right]+\frac{\tau_{0} \sin \phi}{20 \rho H a^{2} \mu} r \mathrm{e}^{-a r}\left[13 \frac{a}{\alpha}+\frac{13}{\alpha^{2}}-\alpha^{2}\right] .
$$

Figure A2 shows contour plots of (A.3), normalised by the factor $\tau_{0} a \mathrm{e}^{-a r} /(20 \rho h \mu)$, for two values of $\alpha$. A pair of anti-symmetric gyres in which the flow is geostrophically balanced is 
observed in both plots. The absence of the Sverdrup balance on the $f$-plane gives rise to the mode one radial gyre structure.
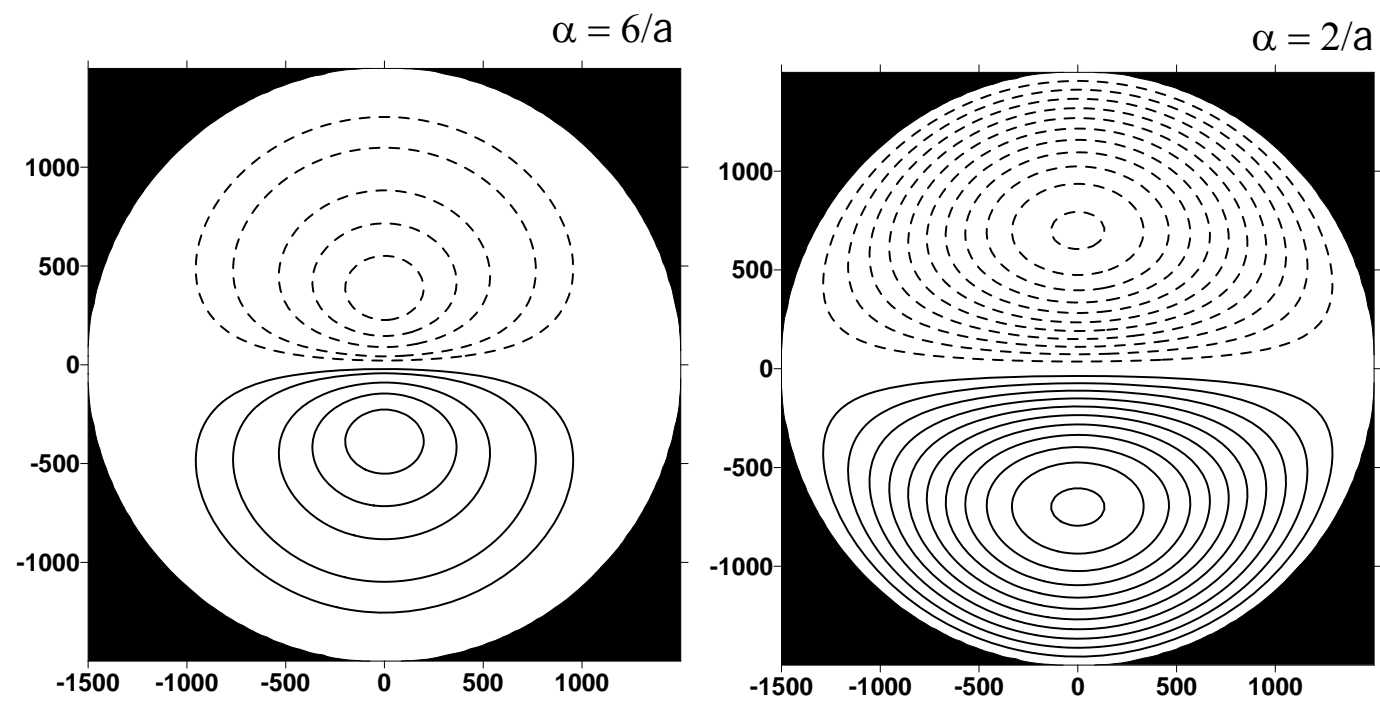

Figure A2. Contour plot of the dimensionless velocity streamfunction (A.3) on an $f$-plane, where; (a) $\alpha^{-1}=a / 6$; (b) $\alpha^{-1}=a / 2$ for comparison with the polar-plane plots in figure 4(a) and (d) respectively. Contour interval is 0.05 . 